\title{
Häusliche Krankenpflege: Neues Formular!
}

_ Ab dem 1. Oktober 2017 wird das Verordnungsformular für die häusliche Krankenpflege, das Muster 12, generalüberholt. Neben den bisherigen Maßnahmen kann dann auch die vor einem Jahr eingeführte „Unterstützungspflege" mit dem Formular verordnet werden.

Darüber hinaus wird das Ausfüllen erleichtert. So ist eine gesonderte Begründung bei einer Verordnungsdauer von über 14 Tagen künftig nicht mehr nötig. Auch muss die Dauer der Medikamentengabe nur noch dann gesondert angegeben werden, wenn der Patient ein Arzneimittel nicht während des gesamten Zeitraums der häuslichen Krankenpflege einnehmen soll. Leistungen wie Medikamentengabe, Blutzuckermessung, Kompressionsbehandlung oder Wundversorgung werden gezielt abgefragt. Leistungen, die seltener vorkommen, können unter „Sonstige Maßnahmen“ als Freitext angegeben werden.

\section{MMW-KOMMENTAR}

Die wichtigste Ergänzung ist ein simples Ankreuzfeld für die Verordnung von

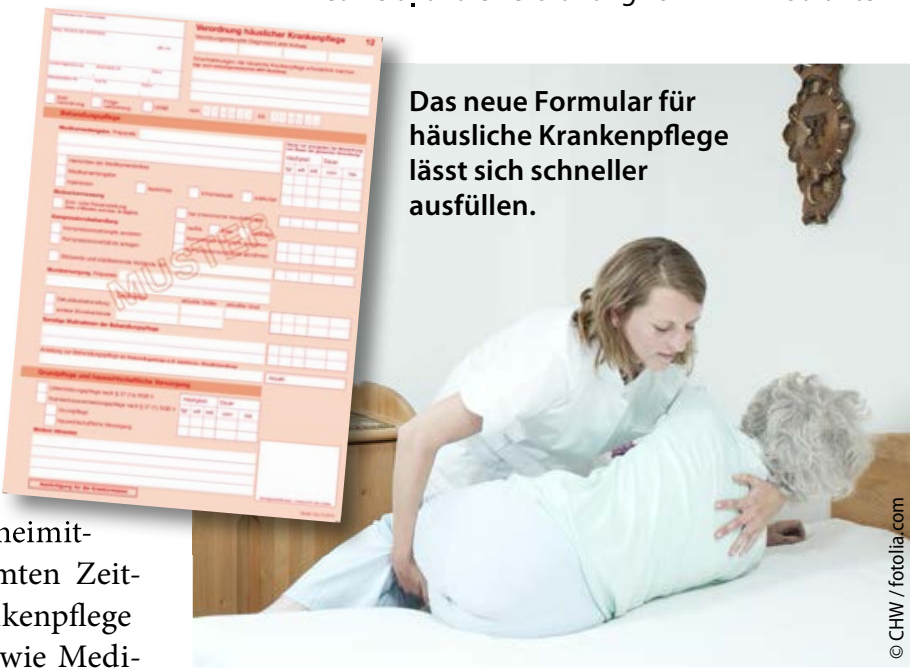

Grundpflege und hauswirtschaftlicher Versorgung nach § 37 Abs. 1a SGB V. Diese sogenannte Unterstützungspflege wurde bereits
2016 in den GKV-Leistungskatalog aufgenommen. Sie kann wegen schwerer Krankheit oder akuter Krankheitsverschlechterung, insbesondere nach einem Klinikaufenthalt, einer ambulanten Operation oder einer ambulanten Krankenhausbehandlung, verordnet werden, sofern der Patient nicht nach SGB XI pflegebedürftig ist.

Ferner entfällt der Hinweis, dass kein Anspruch auf häusliche Krankenpflege besteht, wenn der Versicherte oder eine im Haushalt lebende Person die erforderlichen Maßnahmen übernehmen könnte. Der Arzt muss daher auch nicht mehr ankreuzen, dass er nicht beurteilen kann, ob dieser Hinderungsgrund vorliegt. Ab 1. Oktober 2017 gilt nur noch das neue Muster 12, die alten Formulare darf man

dann nicht mehr nutzen. Das neue Muster wird für den Direktdruck auch in den Praxisverwaltungssystemen hinterlegt.

\section{Hier steht eine Anzeige.}

